\title{
ANÁLISE DO SICK SCAPULA EM JOGADORES \\ DE HANDEBOL COM E SEM DOR NO \\ OMBRO DURANTE O ARREMESSO
}

\author{
ANALYSIS OF THE SICK SCAPULA IN HANDBALL PLAYERS WITH AND WITHOUT \\ SHOULDER PAIN DURING THROWING \\ ANÁLISIS DEL SICK SCAPULA EN JUGADORES DE HANDBOL CON Y SIN DOLOR \\ EN EL HOMBRO DURANTE EL LANZAMIENTO
}

\begin{abstract}
Gabriel Peixoto Leão Almeida ${ }^{1,2}$ (Fisioterapeuta)

Paula Fiquetti Silveira'

(Fisioterapeuta)

Nathália Polisello Rosseto ${ }^{1}$

(Fisioterapeuta)

Gisele Barbosa' (Fisioterapeuta)

Benno Ejnisman' (Médico)

Moisés Cohen ${ }^{1}$ (Médico)

1. Universidade Federal de São

Paulo (DOT/Unifesp), São Paulo,

SP, Brasil.

2. Universidade Federal do Ceará

(UFC), Fortaleza, CE, Brasil.
\end{abstract}

\section{Correspondência:}

Rua Alexandre Baraúna, 949

10 andar, Rodolfo Teófilo

CEP 60430-160, Fortaleza, Ceará,

Brasil. gabriel_alm@hotmail.com

\section{RESUMO}

Introdução: A discinesia escapular é definida como uma alteração da posição escapular, tanto dinâmica quanto estática, resultante de desequilíbrios da musculatura periescapular secundários à fadiga, trauma ou lesão neurológica. O SICK Scapula avalia e caracteriza as alterações escapulares estaticamente, variando de 0 a 20 pontos $(0=$ melhor possível). No exame, a escápula é avaliada em três aspectos: dor objetiva, dor subjetiva e mau posicionamento escapular. Objetivo: Comparar o SICK Scapula entre jogadores de handebol sintomáticos e assintomáticos. Métodos: A amostra foi composta por 57 atletas de handebol divididos em dois grupos, de acordo com a presença de dor no ombro: grupo assintomático (GA) (N=27) e grupo sintomático (GS) ( $N=30)$. O SICK Scapula foi avaliado entre os atletas, tanto em relação à sua pontuação final como às subescalas. O GS apresentou maior pontuação no SICK Scapula em relação ao GA $(8 \pm 2,3$ vs $2,7 \pm 1,8 ; p<0,001)$. Resultados: Nas subescalas, os GA e GS também apresentaram diferenças significantes quanto à dor subjetiva ( 0 vs. $1,73 \pm 0,83 ; p<0,001)$, dor objetiva $(0,41 \pm 0,64$ vs. $2,5 \pm 0,86 ; p<0,001)$ e mau posicionamento escapular $(2,3 \pm 1,9$ vs. 3,7 $\pm 1,5 ; p=0,002)$. Conclusão: Os atletas de handebol com dor relacionada ao arremesso apresentam maior pontuação com relação à dor e às alterações de posicionamento escapular, segundo avaliação pelo SICK Scapula, em comparação com os que não apresentam sintomatologia.

Palavras-chave: dor de ombro, escápula, discinesias, medicina esportiva, atletas.

\section{ABSTRACT}

Introduction: The scapular dyskinesia is defined as a change in scapular position, both dynamic and static, resulting from periscapular imbalances secondary to muscle fatigue, trauma or neurological injury. The SICK Scapula statically evaluates and characterizes the scapular changes, ranging from 0 to $20(0=b e s t)$. This exam addressed three aspects: objective pain, subjective pain and scapular malposition. Objective: To compare the SICK Scapula in symptomatic and asymptomatic handball players. Methods: The sample consisted of 57 handball athletes divided into two groups according to the presence of shoulder pain: asymptomatic group $(A G)(N=27)$ and symptomatic group (SG) $(N=30)$. The SICK Scapula score has been reported among athletes, both in relation to the total score and its subscales. The GS had a significantly higher score than the GA $(8 \pm 2.3$ vs. $2.7 \pm 1.8 ; p<0.001)$. Results: In the subscales, GA and SG also showed significant differences in subjective pain ( 0 vs. 1.73 $\pm 0.83, p<0.001)$, objective pain ( $0.41 \pm 0.64$ vs. $2.5 \pm 0.86, p<0.001)$ and scapular malposition $(2.3 \pm 1.9$ vs. $3.7 \pm 1.5, p=0.002)$. Conclusion: The handball athletes with throwing-related pain have a higher score with respect to pain and changes of scapular positioning, as assessed by SICK Scapula, compared with those who did not have symptoms.

Keywords: shoulder pain, scapula, dyskinesias, sports medicine, athletes.

\section{RESUMEN}

Introducción: La discinesia escapular es definida como una alteración de la posición escapular, tanto dinámica como estática, resultante de desequilibrios de la musculatura periescapular secundarios a la fatiga, trauma o lesión neurológica. EI SICK Scapula evalúa y caracteriza las alteraciones escapulares estáticamente, variando de 0 a 20 puntos $(0=$ mejor posible). En el examen, la escápula es evaluada en tres aspectos: dolor objetivo, dolor subjetivo y mal posicionamiento escapular. Objetivo: Comparar el SICK Scapula entre jugadores de handbol sintomáticos y asintomáticos. Métodos: La muestra fue compuesta por 57 atletas de handbol divididos en dos grupos, de acuerdo con la presencia de dolor en el hombro: grupo asintomático (GA) ( $N=27)$ y grupo sintomático (GS) (N=30). EI SICK Scapula fue evaluado entre los atletas, tanto en relación a su puntuación final como a las subescalas. GS presentó mayor puntuación en el SICK Scapula en relación al GA (8 2,3 vs 2,7 $\pm 1,8 ; p<0,001)$. Resultados: En las subescalas, los GAy GS también presentaron diferencias significativas sobre el Dolor Subjetivo ( 0 vs. 1,73 $\pm 0,83 ; p<0,001$ ), Dolor Objetivo $(0,41 \pm 0,64$ vs. $2,5 \pm 0,86 ; p<0,001)$ y mal posicionamiento escapular $(2,3 \pm 1,9$ vs. 3,7 $\pm 1,5 ; p=0,002)$. Conclusión: 
Los atletas de handbol con dolor relacionado al lanzamiento presentan mayor puntuación con relación al dolor y a las alteraciones de posicionamiento escapular, según evaluación por el SICK Scapula, en comparación con los que no presentan sintomatología.

Palabras clave: dolor de hombro, escápula, discinesias, medicina deportiva, atletas.

\section{INTRODUÇÃO}

Jogadores de handebol realizam cerca de 48 mil movimentos de arremesso no ombro durante uma temporada, com uma bola pesando cerca de 425 a $475 \mathrm{~g}$ e em uma velocidade média de $130 \mathrm{~km} / \mathrm{h}^{1,2}$. Somando o alto estresse repetitivo com a velocidade elevada, os jogadores de handebol são susceptíveis a alterações adaptativas no ombro arremessador levando um aumento de rotação lateral e diminuição da rotação medial em relação ao membro não arremessador ${ }^{3,4}$. Duas hipóteses principais são descritas na literatura como possíveis causas dessas adaptações: a primeira relata que ocorre uma contratura da capsula posterior e da banda posterior do ligamento glenoumeral inferior causados pelo microtrauma repetitivo durante a fase de desaceleração do arremesso, quando ocorre essa contratura, o centro de rotação do úmero desloca na direção pósterosuperior, diminuindo o ponto de contato da cabeça do úmero com o aspecto antero-inferior da capsula ${ }^{5,6}$; a segunda hipótese refere que devido o movimento repetitivo de arremesso ocorrem adaptações ósseas levando a uma retroversão da cabeça do úmero, gerando um déficit de rotação interna do ombro que é considerado o fator primário no desenvolvimento de lesões no ombro do arremessador ${ }^{1,3}$.

Associado a diminuição de amplitude de rotação interna, alguns autores têm sugerido que a disfunção escapular pode ter um papel significante nas patologias do ombro arremessador ${ }^{7,8}$. A discinesia escapular é definida como uma alteração na posição escapular, tanto dinâmica quanto estática, resultante de desequilíbrios da musculatura periescapular secundário à fadiga, trauma ou lesão neurológica9,10. Tem sido relacionadas com diminuição do desempenho no arremesso e com o aumento na incidência de lesões do membro superior dos arremessadores ${ }^{7-9,11}$.

Burkhart et al. ${ }^{7}$, em 2003, publicaram uma série de trabalhos no qual denominaram a síndrome da discinesia escapular como "SICK Scapula". O SICK Scapula faz referência ao Scapular malposition (alteração no posicionamento escapular), Inferior medial border prominence (proeminência do bordo medial da escápula), coracoid pain and malposition (dor e alteração do posicionamento do processo coracoide), dyskinesis (discinesia) do movimento escapular?.

Embora muitos autores tenham procurado mostrar através de análises cinemáticas esses tipos de alterações na escápula 8,12-15, durante a prática clínica nem sempre é possível dispor de tais recursos de avaliação. O exame específico da escápula elaborado por Burkhart et al. ${ }^{7}$ é caracterizado como: SICK Scapula Rating Scale: Static Measurements (0-20 POINTS) que avalia e caracteriza as alterações escapulares estaticamente.

Para nosso conhecimento, este é o primeiro estudo que utiliza o SICK Scapula como medida de avaliação em atletas com e sem dor no ombro durante o arremesso. Portanto, o objetivo deste estudo foi comparar o SICK Scapula entre jogadores de handebol com e sem dor no ombro durante o arremesso.

\section{MATERIAIS E MÉTODOS}

A presente pesquisa trata-se de um estudo transversal caso- Controle, no qual participaram 64 indivíduos (36 homens e 28 mulheres), com faixa etária entre 18 e 37 anos de idade. A seleção e coleta de dados foram realizadas na Universidade Federal de São Paulo (DOT) Unifesp), São Paulo, SP, Brasil. Todos os atletas foram avaliados antes dos treinos e foi solicitado que não realizassem treino ou exercício no dia anterior a avaliação. Para inclusão no grupo assintomático (GA), todos os atletas deveriam ser federados, ter tempo de prática de no mínimo 2 anos, treinar pelo menos 3 vezes por semana e $2 \mathrm{~h}$ por dia, sem história de dor no ombro nos últimos 3 meses e referir zero na Escala Visual Análoga de Dor (EVA; 0 = ausência de dor, 10 = pior dor imaginável). Para inclusão no grupo sintomático (GS), os critérios foram os mesmos para o GA, porém deveriam relatar dor no ombro arremessador por no mínimo 1 mês e dor reproduzida durante o arremesso com pontuação de no mínimo 3 na EVA. Os critérios de exclusão para ambos os grupos foram fratura, luxação ou cirurgia no complexo do ombro e cotovelo, doenças relacionadas à coluna cervical e dorsal, dor no ombro não arremessador, uso de anabolizantes e os que estivessem realizando tratamento fisioterápico ou medicamentoso nos últimos 30 dias. Sete atletas foram excluídos (4 por tratamento fisioterápico ou medicamentoso; 2 por cirurgia prévia no ombro; 1 por uso de anabolizantes), totalizando no final 27 atletas no GA e 30 no GS.

Antes de iniciar a pesquisa, todos os indivíduos foram informados sobre os procedimentos do estudo e assinaram um Termo de Consentimento Livre e Esclarecido. A presente pesquisa foi aprovada pelo Comitê de Ética e Pesquisa da Universidade Federal de São Paulo (DOT/ Unifesp), São Paulo, SP, Brasil.

Cada participante respondeu um questionário referente a características antropométricas, nível competitivo, ombro dominante e presença de dor no ombro arremessador. Para determinar o membro arremessador de cada atleta foi dada a seguinte instrução: "Imagine uma bola na sua mão, com qual braço você arremessaria para alcançar a maior força e precisão possível?". Todos os participantes foram submetidos a análise escapular por meio da escala de pontuação do SICK SCAPULA. Os avaliadores responsáveis por essa avaliação foram blindados quanto ao grupo de cada atleta.

Escala de Pontuação SICK Scapula: O protocolo de avaliação escapular foi de acordo com Burkhart et al. ${ }^{7}$. Esta escala é baseada na comparação das duas escápulas podendo variar de 0 (melhor possível) a 20 (pior possível) pontos e consiste da soma dos seguintes itens: Dor Subjetiva ( 0 - 5 pontos), Dor Objetiva ( 0 - 6 pontos) e Mau Posicionamento Escapular (0 - 9 pontos). O Mau Posicionamento Escapular foi inicialmente avaliado com o atleta em pé e com os membros superiores ao longo do corpo. Nesta posição, as diferenças entre as escápulas foram determinadas pela altura (em centímetros) do ângulo superior da escápula, a distância (em centímetros) do ângulo superior da escápula para o processo espinhoso no mesmo nível e a diferença angular (em graus) do bordo medial da escápula em relação a uma linha reta perpendicular ao solo (figura 1). Para Dor Subjetiva, o atleta respondia "Sim" ou "Não" se sentia dor no Processo Coracóide, Articulação Acromioclavicular, Região Periescapular, Região Lateral e Proximal do Braço e se sentia Sintomas Radiculares no membro superior. Por último, para dor objetiva cada atleta respondia "Sim" ou "Não"em relação à dor na palpação do processo coracóide, articulação acromioclavicular e ângulo superior da escápula, dor reproduzida no teste de impacto 


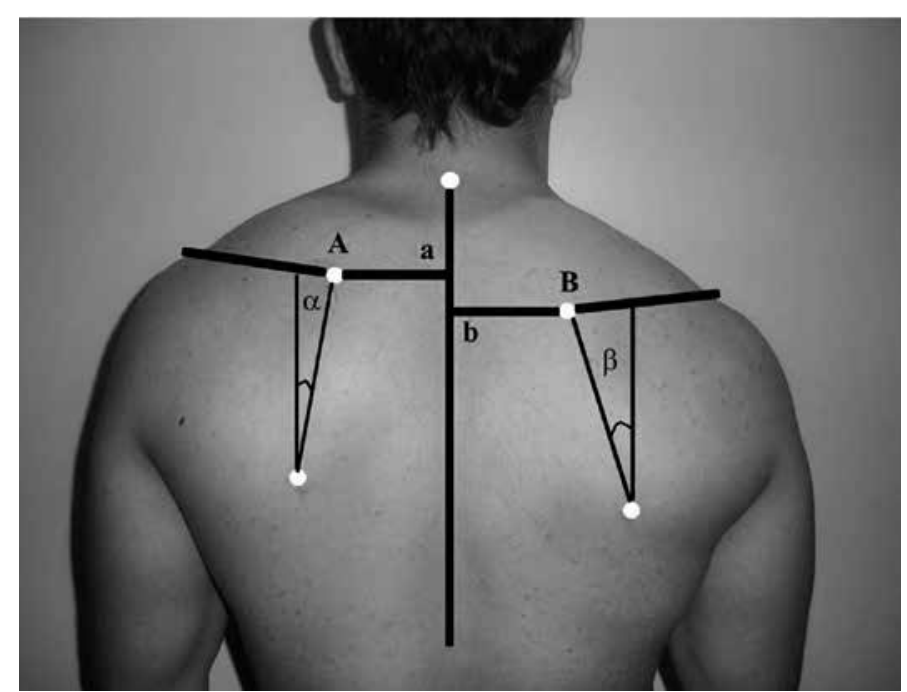

Figura 1. Comparações entre as escápulas. (A,B), diferença na altura do ângulo superior da escápula; (a,b), diferença na distância do ângulo superior as escápula para o processo espinhoso do mesmo nível. $(\alpha, \beta)$, diferença do ângulo formado entre o bordo medial da escapula e uma linha perpendicular ao solo.

(Kennedy-Hawkins), nos testes para Síndrome do Desfiladeiro Torácico (Teste de Adson, Éden e Wright) e diminuição dos sintomas no Teste de Assistência Escapular. As diferenças de 0 - 3 centímetros correspondem de $0-3$ pontos respectivamente, e as diferenças de $0^{\circ}, 5^{\circ}, 10^{\circ} \mathrm{e} 15^{\circ}$ correspondem 0, 1, 2 e 3 pontos, respectivamente.

\section{Estudo Piloto}

A confiabilidade intra-avaliador foi estimada antes do início da coleta dos dados por meio do Coeficiente de Correlação Intraclasse $\left(\mathrm{CCl}_{3,1}\right)$. Para isso, 12 jogadores de handebol, sendo seis com dor no ombro, com idade de $21,5 \pm 2,8$ anos, altura 1,73 $\pm 0,08 \mathrm{~m}$ e peso 66,2 $\pm 6,9 \mathrm{~kg}$ foram submetidos a avaliação do SICK Scapula de acordo com o protocolo descrito acima em dois momentos, com intervalo de uma semana entre a primeira e segunda avaliação. Excelente confiabilidade foi demonstrada para o SICK Scapula com CCl $\mathrm{Cl}_{3,1}$ de 0,91. Para suas subescalas, a confiabilidade da Dor Subjetiva foi 0,93, Dor Objetiva foi 0,92, e o Mau Posicionamento Escapular apresentou confiabilidade de 0,78.

\section{Análise Estatística}

Inicialmente avaliou-se a normalidade dos dados pelo teste de Kolmogorov-Smirnov. O teste qui-quadrado $\left(x^{2}\right)$ foi utilizado para identificar diferenças entre os grupos em relação ao sexo. $O$ teste $t$ -independente foi utilizado para verificar as diferenças em relação a idade, peso, altura, tempo de prática esportiva e horas de treino por semana. Por último, o teste de Mann-Whitney foi usado para identificar possíveis diferenças entre os dois grupos quanto ao SICK Scapula e suas subescalas. Todas as análises estatísticas foram realizadas com o software SPSS 17.0 para Windows (Statistical Package for the Social Sciences Inc., Chicago, IL, USA).

\section{RESULTADOS}

Os grupos não apresentaram diferenças significantes quanto à idade, sexo, peso, altura, tempo de prática e horas de treino por semana (tabela 1). O GS apresentou média de 4,83 $\pm 1,2$ pontos na EVA.

Quanto às comparações do SICK Scapula, o GS apresentou diferenças estatisticamente significantesem relaçãoGA ( $8 \pm 2,3$ vs $2,7 \pm 1,8$; $\mathrm{p}<0,001)$. Nas subescalas, os GA e GS também apresentaram diferenças significantes quanto a Dor Subjetiva (0 vs $1,73 \pm 0,83 ; p<0,001)$, Dor Objetiva $(0,41 \pm 0,64$ vs $2,5 \pm 0,86 ; p<0,001)$ e Mau Posicionamento Escapular (2,3 $\pm 1,9$ vs $3,7 \pm 1,5 ; \mathrm{p}=0,002)$ (figura 2).
Tabela 1. Comparação entre o Grupo Sintomático (GS) e o Grupo Assintomático (GA) em relação a variáveis antropométricas e características esportivas (média e desvio padrão).

\begin{tabular}{c|c|c|c}
\hline & GS $(\mathbf{n}=\mathbf{3 0})$ & $\mathbf{G A}(\mathbf{n}=\mathbf{2 7})$ & Valor Estatístico \\
\hline Sexo (M:F) & $15: 15$ & $13: 14$ & $\mathrm{X}^{2}=, 019, \mathrm{P}=0,89$ \\
\hline Idade (anos) & $20,5 \pm 2,5$ & $19,8 \pm 2,1$ & $\mathrm{t}=-1,15, \mathrm{P}=0,25$ \\
\hline Peso (Kg) & $69,4 \pm 10,1$ & $68,3 \pm 11,8$ & $\mathrm{t}=-, 368, \mathrm{P}=0,71$ \\
\hline Altura (cm) & $1,76 \pm 0,1$ & $1,77 \pm 0,1$ & $\mathrm{t}=, 368, \mathrm{P}=0,71$ \\
\hline $\begin{array}{c}\text { Tempo de prática } \\
\text { esportiva }\end{array}$ & $6,9 \pm 2,9$ & $6,3 \pm 2,6$ & $\mathrm{t}=-, 771, \mathrm{P}=0,44$ \\
\hline $\begin{array}{c}\text { Horas de treino por } \\
\text { semana }\end{array}$ & $11,6 \pm 3,4$ & $11,4 \pm 4,8$ & $\mathrm{t}=-, 141, \mathrm{P}=0,89$ \\
\hline
\end{tabular}

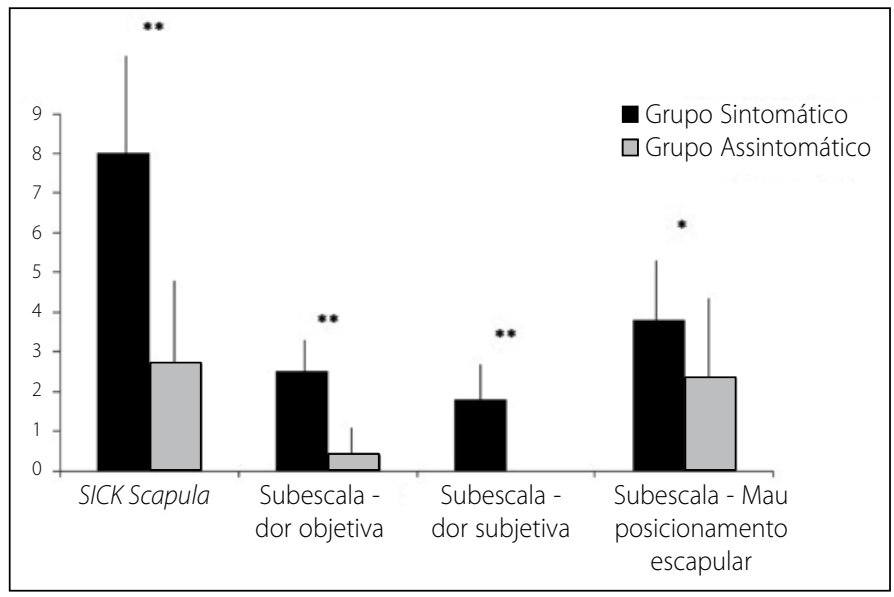

Figura 2. Diferenças entre os grupos quando a pontuação do SICK Scapula e suas subescalas. ${ }^{*} p<0,01 ;{ }^{* *} p<0,001$.

\section{DISCUSSÃO}

A presente pesquisa demonstrou que atletas de handebol que relataram dor no ombro durante o arremesso apresentaram maior pontuação no SICK Scapula e suas subescalas em comparação aos atletas sem dor. O ombro é a região anatômica com maior incidência de lesões por esforço repetitivo nos atletas de handebol, estudos demonstram que a taxa de frequência das lesões agudas e crônicas no ombro desses atletas varia de 30 - $45 \% \%^{1,2}$. O posicionamento adequado e o movimento apropriado da escápula permitem que os atletas possam atingir o máximo de desempenho com menos riscos de lesões no ombro $8,10,16$. Durante o movimento de arremesso com o braço em posição de $90^{\circ}$ de abdução e $90^{\circ}$ de flexão do cotovelo, a escápula gira superiormente ${ }^{12,15,16}$, realiza uma rotação externa e inclina posteriormente ${ }^{8,10,16}$, dessa forma aumenta o espaço subacromial ${ }^{17,18}$ e otimiza a transmissão de força seqüencial de proximal para distal permitindo maior energia, velocidade e força no arremesso 17,19,20.

As alterações na cinemática escapular em arremessadores são descritas na literatura tanto em atletas com lesão no ombro como sem lesão. As adaptações naturais na dinâmica escapular pode sem identificadas nos estudos de Myes et al. ${ }^{15}$, que relatam que atletas arremessadores apresentaram aumento da rotação superior, rotação interna e retração da escapula em comparação com indivíduos não arremessadores, e Downar et al. ${ }^{12}$ que descreveram um aumento da rotação superior da escápula no membro arremessador em comparação ao não arremessador. Porém, aqueles atletas que apresentam dor no ombro possuem maiores alterações na cinemática escapular que os atletas assintomáticos. Isso é comprovado com estudos de Laudner 
et al. ${ }^{16}$ que relatam que atletas arremessadores com diagnóstico de impacto interno do ombro apresentaram maior inclinação posterior da escapula comparado a arremessadores lesão, e Su et al. ${ }^{21}$ que compararam 20 nadadores com síndrome do impacto com 20 nadadores assintomáticos, e encontraram uma diminuição na rotação superior no grupo com impacto após a prática de natação.

Diferente dos estudos citados acima, que utilizaram em suas pesquisas métodos de avaliação 3D e inclinômetros digitais, a escala de pontuação SICK Scapula considera as alterações de posicionamento escapular de maneira estática quando a mesma está aparentemente inferior, em translação lateral e abdução (perda do controle da protração) em comparação ao membro contralateral ${ }^{7}$. Na presente pesquisa, o grupo com dor apresentou pontuação significativamente maior no SICK Scapula e suas subescala sem comparação ao grupo sem dor (figura 2). Infelizmente, não foram encontrados outros artigos que avaliassem a escápula de forma parecida, impossibilitando comparações desses resultados com outras publicações. No conhecimento dos autores, apenas um artigo 22 utilizou o SICK Scapula na sua metodologia para avaliar pacientes com luxação acromioclavicular tipo III, e encontraram uma média de 6,9 pontos. Eles determinaram que se o paciente obtiver score menor que 5 pontos, as atividades de arremesso sobre a cabeça não estão limitadas, de 5 à 10 pontos, tais atividades são dolorosas mas possíveis, e quando atingissem scores maiores que 10 pontos não poderiam abduzir ou elevar o braço mais que $90^{\circ}$ e ainda teriam dor em repouso. Esta classificação proposta por Gumina et al. ${ }^{22}$ pode ser verificada na presente pesquisa, no qual o grupo assintomático ficou com média de 2,7 pontos e o sintomático com média de 8 pontos.

O SICK Scapula é um método de avaliação da escápula desenvolvido principalmente para atletas arremessadores. Essa medida leva bastante em consideração a dor dos atletas, já que, de suas três subescalas, duas são relacionadas a dor e apenas uma relacionada a alterações do posicionamento escapular, sendo ainda analisada de forma estática. Não é surpreendente que os atletas com dor no ombro apresentem maior pontuação nas subescalas de dor objetiva e subjetiva, mas é interessante o fato de ter sido visualizado alterações no posicionamento escapular.

A presente pesquisa trouxe dados interessantes, porém possui algumas limitações. (1) A natureza transversal da pesquisa impossibilita determinar se as alterações no posicionamento escapular no GS foi um fator de risco para o desenvolvimento de dor no ombro ou a consequência. (2) A subjetividade da classificação dos grupos (presença ou ausência de dor no arremesso) sem um diagnóstico clínico por meio de testes ou exames complementares também é uma limitação importante do presente estudo. (3) Futuras pesquisas poderiam relacionar o SICK Scapula com avaliação dinâmica da escápula em 3D, fornecendo maior validade para este método de avaliação escapular.

\section{CONCLUSÃO}

Atletas de handebol com dor no ombro apresentaram maior pontuação no SICK Scapula e em suas subescalas sem relação aqueles sem dor. Devido à natureza transversal do estudo, estes achados não permitem determinar a causa-efeito do mau posicionamento escapular, porém demonstram a importância da avaliação da escápula em arremessadores possibilitando identificar alterações e instituir o melhor tratamento precocemente.

Todos os autores declararam não haver qualquer potencial conflito de interesses referente a este artigo.

\section{REFERÊNCIAS}

1. Pieper HG. Humeral torsion in the throwing arm of handball players. Am J Sports Med. 1998;26(2):247-53.

2. Seil R, Rupp S, Tempelhof S, Kohn D. Sports injuries in team handball. A one-year prospective study of sixteen men's senior teams of a superior nonprofessional level. Am J Sports Med. 1998;26(5):681-7.

3. Wilk KE, Macrina LC, Fleisig GS, Porterfield R, Simpson CD 2nd, Harker P, et al. Correlation of glenohumeral internal rotation deficit and total rotational motion to shoulder injuries in professional baseball pitchers. Am J Sports Med. 2011;39(2):329-35.

4. Whiteley R, Ginn K, Nicholson L, Adams R. Indirect ultrasound measurement of humeral torsion in adolescent baseball players and non-athletic adults: reliability and significance. J Sci Med Sport. 2006;9(4):310-8.

5. Reagan KM, Meister K, Horodyski MB, Werner DW, Carruthers C, Wilk K. Humeral retroversion and its relationship to glenohumeral rotation in the shoulder of college baseball players. Am J Sports Med. 2002;30(3):354-60.

6. Thomas SJ, Swanik CB, Kaminski TW, Higginson JS, Swanik KA, Bartolozzi AR, et al. Humeral retroversion and its association with posterior capsule thickness in collegiate baseball players. J Shoulder Elbow Surg. 2012;21(7):910-6

7. Burkhart SS, Morgan CD, KiblerWB. The disabled throwing shoulder: spectrum of pathology Part III: The SICK scapula, scapular dyskinesis, the kinetic chain, and rehabilitation. Arthroscopy. 2003;19(6):641-61.

8. Kibler WB, Sciascia A. Current concepts: scapular dyskinesis. Br J Sports Med. 2010;44(5):300-5.

9. Ludewig PM, Reynolds JF. The association of scapular kinematics and glenohumeral joint pathologies. J Orthop Sports Phys Ther. 2009;39(2):90-104.

10. Cools AM, Johansson FR, Cambier DC, Velde AV, Palmans T, Witvrouw EE. Descriptive profile of scapulothoracic position, strength and flexibility variables in adolescent elite tennis players. Br J Sports Med. 2010;44(9):678-84.

11. Cools AM, Witvrouw EE, Declercq GA, Vanderstraeten GG, Cambier DC. Evaluation of isokinetic force production and associated muscle activity in the scapular rotators during a protraction-retraction movement in overhead athletes with impingement symptoms. Br J Sports Med. 2004;38(1):64-8.

12. Downar JM, Sauers EL. Clinical Measures of Shoulder Mobility in the Professional Baseball Player. J Athl Train. 2005;40(1):23-9.

13. Ebaugh DD, McClure PW, Karduna AR. Effects of shoulder muscle fatigue caused by repetitive overhead activities on scapulothoracic and glenohumeral kinematics. J Electromyogr Kinesiol. 2006;16(3):224-35.

14. Ebaugh DD, McClure PW, Karduna AR. Scapulothoracic and glenohumeral kinematics following an external rotation fatigue protocol. J Orthop Sports Phys Ther. 2006;36(8):557-71.

15. Myers JB, Laudner KG, Pasquale MR, Bradley JP, Lephart SM. Scapular position and orientation in throwing athletes. Am J Sports Med. 2005;33(2):263-71.

16. Laudner KG, Myers JB, Pasquale MR, Bradley JP, Lephart SM. Scapular dysfunction in throwers with pathologic internal impingement. J Orthop Sports Phys Ther. 2006;36(7):485-94.

17. Meister K. Injuries to the shoulder in the throwing athlete. Part one: Biomechanics/pathophysiology/ classification of injury. Am J Sports Med. 2000;28(2):265-75.

18. McClure PW, Michener LA, Sennett BJ, Karduna AR. Direct 3-dimensional measurement of scapular kinematics during dynamic movements in vivo. J Shoulder Elbow Surg. 2001;10(3):269-77.

19. Escamilla RF, Andrews JR. Shoulder muscle recruitment patterns and related biomechanics during upper extremity sports. Sports Med. 2009;39(7):569-90.

20. Hirashima M, Kadota H, Sakurai S, Kudo K, Ohtsuki T. Sequential muscle activity and its functional role in the upper extremity and trunk during overarm throwing. J Sports Sci. 2002;20(4):301-10.

21. Su KP, Johnson MP, Gracely EJ, Karduna AR. Scapular rotation in swimmers with and without impingement syndrome: practice effects. Med Sci Sports Exerc. 2004;36(7):1117-23.

22. Gumina S, Carbone S, Postacchini F. Scapular dyskinesis and SICK scapula syndrome in patients with chronic type III acromioclavicular dislocation. Arthroscopy. 2009;25(1):40-5. 